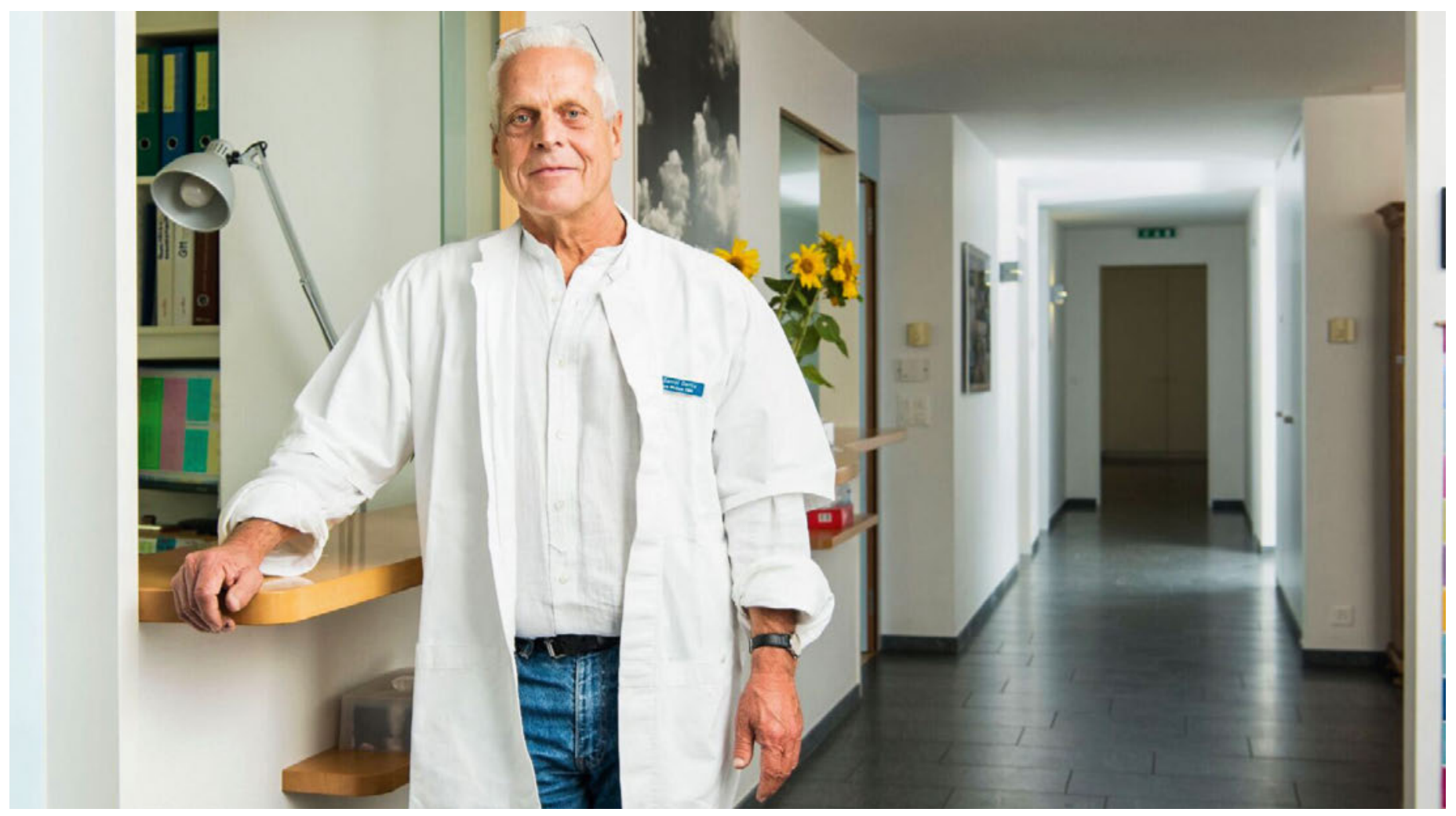

\title{
«Lehren und Lernen ist immer gegenseitig»
}

Inteview: Bruno Schmucki

Verantwortlicher Kommunikation SGAIM

Am Herbstkongress der SGAIM wurde der Zürcher Hausarzt Daniel Oertle für seine langjährige Lehrtätigkeit mit dem «Teaching Award 2017» ausgezeichnet. Im Interview erklärt er, was ihn für dieses Engagement motiviert und warum es wichtig ist, dass gerade auch Hausärzte in der Aus- und Weiterbildung aktiv sind.

Sie wurden soeben mit dem dem «Teaching Award» der SGAIM ausgezeichnet. Was macht in Ihren Augen einen guten Lehrer aus?

Medizin ist Wissenschaft und Kunst: Der wissenschaftliche Teil der Medizin ist das codierbare Wissen, das in Algorithmen verpackt Suchmaschinen zugänglich ist.

«Ein guter Lehrer weiss nicht alles, aber er ist neugierig und will möglichst viel wissen."

In Zeiten von Google, Wikipedia und Uptodate hat der Lehrer kein Wissensmonopol mehr; jeder trägt auf sei- nem Smartphone das ganze Wissen im Hosensack und gerade die jungen Leute bewegen sich in diesem Universum sehr flink und schlau.

Ein guter Lehrer weiss nicht alles, aber er ist neugierig und will möglichst viel wissen. Er steckt die Lernenden mit seiner Neugierde und Wissbegierde an und gemeinsam gehen Lehrer und Lernende auf die Suche nach dem Wissen.

Medizin ist Wissenschaft und Kunst: Der gute Lehrer lehrt den Schüler die Heilkunst. Der Lehrer leitet den Lernenden an, den Zugang zum Patienten zu finden und ihn in seiner Einzigartigkeit zu erkennen. Der Leh- 


\section{Zur Person:}

Seit 22 Jahren betreibt Dr. med. Daniel Oertle eine Arztpraxis an der Dennlerstrasse in Zürich und zwar in unmittelbarer Nähe des Letzigrundstadions. Die Doppelpraxis zusammen mit Dr. Bruno Maggi ist eine im Quartier gut verankerte und auf den ersten Blick ganz normale Hausarztpraxis.

Aussergewöhnlich ist aber das breite diagnostische und therapeutische Angebot, das von Manueller Medizin über Röntgen bis zu kleinchirurgischen Eingriffen reicht. Beim Blick aufs Curriculum springt vor allem Daniel Oertles Engagement in Sachen Infektiologie ins Auge: Als langjähriger externer Mitarbeiter bei der Schweizerische HIV-Kohortenstudie SHCS und als ehemaliges Mitglied der Ausserparlamentarischen Kommission für AIDS-Fragen bearbeitet er seit vielen Jahren dieses Themenfeld.

Die Praxis an der Dennlerstrasse ist aber auch ein Ort des Lernens. Diese Tatsache lässt sich leicht anhand von Zahlen ableiten: Bereits 49 Assistent(inn)en sammelten wertvolle Erfahrungen in der Hausarztmedizin und mittlerweile 26 Studierende profitierten vom Teaching im Rahmen einer Unterassistenz. Und es kommen laufend Studierende im dritten Ausbildungsjahr hinzu, welchen Daniel Oertle erste Einblicke in die Hausarzttätigkeit verschafft. Die Ausbildungstätigkeit erfolgt in Zusammenarbeit mit dem Schweizerischen Kollegium für Hausarztmedizin und dem Institut für Hausarztmedizin der Universität Zürich.

rer hört dem Lernenden zu, wie dieser die Anamnese erhebt und zeigt ihm die körperliche Untersuchung. Der gute Lehrer vermittelt dem Lernenden freigebig seine handwerklichen Fertigkeiten.

\section{«Gutes und lustvolles Lernen findet an einem angenehmen Ort statt, im angeregten Mitein- ander eines Teams.»}

\section{Was glauben Sie, schätzen Ihre Studentinnen und Studenten besonders an Ihnen bzw. an Ihrer Lehre?} In unserer Gemeinschafts-Praxis arbeiten zwei Lehrärzte, zwei Assistenzärzte/-ärztinnen, mehrere Medizinische Praxisassistentinnen und MPA-Auszubildende, Studierende und Unterassistent(inn)en. Gutes und lustvolles Lernen findet an einem angenehmen Ort statt, im angeregten Miteinander eines Teams. In dieser Lernatmosphäre kann zum Beispiel die Medizinische Praxisassistentin dem Assistenzarzt beibringen, wie sie bei schwierigen Venenverhältnisse eine Infusion legt. Und der Unterassistent zeigt dem Lehrarzt die Wundversorgungstechnik, die er kürzlich an einer anderen Lehrstelle erlernt hatte, während der Assistenzarzt einen Fallbericht für eine Fachzeitschrift verfasst.

Das gemeinsame Mittagessen in der Praxis ist ein guter Rahmen, um Wissen und Haltungen ungezwungen auszutauschen. examen gemacht. Mein Wissen ist 40 Jahre alt und verstaubt. Hätten wir nicht all die wunderbar motivierenden Studierenden und Assistenten gehabt, die in den letzten 22 Jahren in unserer Praxis ein- und ausgingen, wäre ich auch verstaubt.

\section{Was sind die kommenden Herausforderungen in der Lehre der AIM?}

Die Universität muss ihre Rolle in der Ausbildung der Mediziner neu finden. Die codierbaren wissenschaftlichen Inhalte werden sich die zukünftigen Studierenden rasch und mühelos selber aneignen können. Die Universitäten werden aber die Studierenden in der Medizinkunst anleiten und hier sind sie zwingend auf die niedergelassenen Ärztinnen und Ärzte angewiesen.

\section{Ein Schwerpunkt der SGAIM ist die Nachwuchsförde- rung. Was sind Ihrer Meinung nach die besten Argumente, junge angehende Ärztinnen und Ärzte für die AIM zu gewinnen?}

Nachwuchsförderung beginnt sehr früh. Schauen Sie beim Fussball: Wer in der Champions League spielen will, muss eine eigene Akademie aufbauen, muss bereits die Talente der E-Junioren erkennen und sie fördern und begleiten.

Angehende Mediziner sollen früh und häufig in der Allgemeinpraxis hospitieren und zwingend einen grossen Teil ihrer Ausbildung dort absolvieren. Insbesondere soll die Unterassistenz in der Allgemeinpraxis obligatorisch werden, wobei die Universitäten die Lehrärzte logistisch und finanziell unterstützen.

So können wir den Lernenden die wunderbare Vielfalt unserer Arbeit in der Praxis zeigen und sie für die abwechslungsreiche Allgemeine Innere Medizin begeistern

"Angehende Mediziner sollen früh und häufig in der Allgemeinpraxis hospitieren und zwingend einen grossen Teil ihrer Ausbildung dort absolvieren.»

Ich gebe Ihnen ein Beispiel: 2006 fragte uns ein Student während des "Pöpperlikurses», ob wir auch Unterassistenten in unserer Praxis beschäftigten. Und er wurde 2008 unser erster «Uhu». 2014 absolvierte er ein halbes Jahr seiner Weiterbildung in Allgemeiner Innerer Medizin in unserer Praxis. Seit Sommer 2017 - elf Jahre seit unserer ersten Begegnung - ist der fertig ausgebildete junge Kollege Partner in unserer Gemein schaftspraxis ...

Bildnachweis

zVg von Daniel Oertle 